\title{
Submitted: $\quad$ Cervical stenosis and pregnancy rate after ultrasound Accepted: 01.06.2020 guided cervical dilation in women undergoing saline infusion sonography
}

Published: 15.06.2020

\author{
Rubina Izhar ${ }^{1}$, Samia Husain¹, Muhammad Ahmad Tahir², Sonia Husain ${ }^{1}$ \\ ${ }^{1}$ Department of Gynaecology And Obstetrics, Aziz. Medical Center, Karachi, Pakistan \\ ${ }^{2}$ Aziz Medical Center, Karachi, Pakistan \\ Correspondence: Samia Husain, Department Of Gynaecology And Obstetrics, \\ Aziz Medical Center, Karachi, Pakistan, 3-D, 25/20, Nazimabad No.3, Karachi; \\ tel.:923453120015,e-mail:Samiahusain_Scorpio@hotmail.com
}

DOI: $10.15557 /$ JoU.2020.0019

Keywords

cervical stenosis, conception, ultrasound-guided cervical dilation

\begin{abstract}
Aim: To assess the effect of ultrasound-guided cervical dilation on clinical pregnancy rates in women with cervical stenosis. Methods: A prospective case control study was conducted in women with unexplained infertility. These women underwent saline infusion sonography and were suspected of having cervical stenosis if a 7 French Foley's catheter could not be introduced into the cervical canal. Women with suspected stenosis who agreed to undergo ultrasound guided dilation were included as cases. Those with suspected stenosis who refused dilation were used as controls. Both groups were followed for one year. The primary outcome measure was women who had clinical pregnancy i.e. fetal heart beat present on transvaginal scan during the follow up period. Results: During the study period, 1097 women who underwent SIS were included. A total of $117(10.7 \%)$ patients had cervical stenosis. No statistically significant difference was there in both groups in terms of mean age $(p=0.617)$, mean body mass index $(p=0.598)$, duration of infertility $(p=0.588)$ and type of infertility $(p=0.167)$. However, both groups were significantly different in terms of history for risk factors i.e. endometriosis $(p<0.001)$, prior surgery $(p<0.001)$, polyps $(p=0.004)$ and pelvic inflammatory disease $(p=0.001)$. Eighty-nine women agreed to have dilation and were used as cases and 28 women refused and were used as controls. Of the cases, 70.45\% conceived as compared to $18.5 \%$ of controls. The mean time to conception in months was also significantly lower in cases $(8.10 \pm 3.43$ vs. $11.39 \pm 1.97, p<0.001)$. Conclusions: Our study shows that ultrasound-guided cervical dilation in women with cervical stenosis can lead to improved conception rates.
\end{abstract}

\section{Introduction}

Despite regular sexual intercourse, around $10-15 \%$ of couples fail to conceive ${ }^{(1)}$. Cervical factor is implicated in around $5 \%$ of these couples ${ }^{(2)}$. Cervical factor can also be caused by cervical stenosis, which remains undiagnosed in the usual fertility workup suggested by European Society of Human Reproduction and Embryology (ESHRE) and Royal College of Obstetricians and Gynaecologists $(\mathrm{RCOG})^{(3)}$. This happens because the fertility panel run on couples does not involve evaluation of the uterine cavity.
Trans-cervical access to uterine cavity, like sonohysterogram or hysterosalpingogram, is needed for the investigation of the uterine cavity; therefore, cervical stenosis may go undiagnosed in a woman with insignificant history till intrauterine insemination or embryo transfer in an in vitro fertilization cycle is attempted ${ }^{(4)}$.

Cervical stenosis can be either congenital or acquired. It may lead to hematometra, pyometra or subfertility ${ }^{(5)}$. Difficult embryo transfers are a frequently cited cause of decreased clinical pregnancy rates in in vitro fertilization 
(IVF) cycles $^{(6)}$. It has been suggested that trial transfers under ultrasound guidance improve conception rates. Saline infusion sonography involves the use of soft tip Foley's catheter for the introduction of saline used for cavity distension and checking for free spill ${ }^{(7)}$. Cervical dilation with a Hegar's dilator may be needed prior to introduction of this Foley's catheter in cases of stenosis. Since a stenosis that hinders introduction of a 7 French Foley's may also be relieved after dilation, the couple may conceive after this resistance has been overcome ${ }^{(8)}$. A closed cervix prevents intrauterine deposition of semen during natural breeding, may delay uterine clearance after insemination, leading to intrauterine fluid accumulation and subsequent infertility.

We undertook this study to assess the effect of cervical dilation in women with cervical stenosis on clinical pregnancy rates.

\section{Methods}

Women aged 20 to 39 years with infertility defined as no conception despite 24 months of regular unprotected sexual intercourse were included in the study. Women with history of cervical cancer, congenital abnormality of the cervix, male factor as assessed by World Health Organization (WHO) semen analysis criteria and women with ovulatory disorders were excluded. Also, women who failed to comply with the follow up were excluded. Written informed consent was taken from all women. Those who met the inclusion criteria then underwent saline infusion sonography (SIS) on days 8 and 11 of their menstrual cycle.

Saline infusion sonography was carried out in the outpatient department. For the procedure, a sterile Cusco's speculum was inserted into the vagina and a cervical Foley's catheter of 6 French was inserted through the cervical os in an aseptic manner. In the case of resistance, the cervical canal was dilated using a Hegar's dilator. Before dilation, a cervical block was applied under local anaesthesia. The insertion was attempted again. The bulb of the Foley's was distended with 1-2 $\mathrm{mL}$ of saline, and then the endometrial cavity was distended by instilling normal saline through the cervical Foley's catheter. Detailed method was previously described in the article ${ }^{(7)}$. In cases where cervical dilation failed under local anaesthesia, the procedure was carried out under general anaesthesia and the catheter was retained for 5 days. Antibiotic cover was given in all cases of dilation. All women received clarithromycin (Klaricid) 500 milligrams in the form of tablets: one tablet twelve hourly for 5 days. Foley's was left in cervices that were difficult to dilate and had instrumentation done more than once. To ensure no infection takes place, clarithromycin was given for 5 days according to the microbial resistance patterns seen in the local setup. The duration of treatment was also assessed according to local practice.

A Mindray's DP-2200 scanner with a 5 to $7.5 \mathrm{MHz}$ frequency endovaginal transducer was used for all procedures. All scans were performed by the lead author who is a specialist in ultrasound and fertility and has 10 years of experience $^{(7)}$.
To calculate an adequate sample size we searched the literature for pregnancy rates after cervical dilation. Literature reports that cervical stenosis is seen in $5 \%$ of women with subfertility. It has also been reported that dilation for cervical stenosis increased the odds of clinical pregnancy ( $80 \%$ vs. $28 \%$, respectively). Assuming that the patients are not conceiving due to cervical stenosis, with a power of $80 \%(1-\beta)$ and a one-sided 0.05 risk of type I error ( $\alpha$ ), we needed 11 patients with cervical stenosis in each group to demonstrate a similar rise in pregnancy rate after dilation in this group of patients. We included 117 women with cervical stenosis in the analysis and divided them into groups based on dilation.

\section{Cases and controls}

Women who received cervical dilation under local or general anaesthesia were included as group A (cases). Cases where cervix was stenosed but dilation was also unsuccessful with Foley's were not included in the final analysis. Women who did not consent to dilation functioned as group B (controls). They were followed for the study duration but not offered dilation after the initial enrolment, till the completion of study. Those who requested dilation later on during the study period were also excluded to avoid protocol deviation.

Sexual contact was encouraged and the women were asked to report back if they became pregnant. Transvaginal scan was done to confirm fetal heart beat.

All women were followed for a period of twelve months. The follow up was maintained via telephone calls and repeat transvaginal scans at 3, 6, 9 and 12 months. Those who did not comply with the follow up were not included in the final statistical analysis. The primary outcome measure was women who had clinical pregnancy i.e. fetal heartbeat present on transvaginal scan after they were serum beta hCG positive during the follow up period.

All participants provided informed consent. In lieu of formal ethics committee or formal institutional review board approval, Helsinki's declaration was followed. No subjects were harmed, confidentiality was maintained, and no subject was enrolled in the study without formal informed consent.

The Shapiro-Wilk test was used to assess normality of data distribution. The Quantitative variables, such as age, duration of infertility and duration from treatment to conception were presented by means and standard deviation. The t- test for two independent samples was used to compare means for both groups; those requesting dilation and those who did not. Frequency and percentages were computed for qualitative variables: type of infertility and conception. Chi square test was used to compare the groups.

Survival analysis was performed to evaluate the probability of conceiving in both groups. Curves were compared by means of Mantel-Haenszel log-rank test for categorical variables, which in this case was pregnancy. Analyses were performed using SPSS version 15.0 statistical package (SPSS, Inc.,) with statistical significance denoted by a $p$-value of less than 0.05 . 
Tab. 1. Characteristics of study population

\begin{tabular}{|l|c|}
\hline & Mean +/- Standard deviation or $\boldsymbol{n}$ (\%) \\
\hline Age in years & $28.99 \pm 5.40$ \\
\hline BMI & $27.57 \pm 4.39$ \\
\hline Duration of infertility in years & $3.94 \pm 1.59$ \\
\hline \multicolumn{2}{|l}{ Type of infertility } \\
\hline Primary & $637(58.1 \%)$ \\
\hline Secondary & $460(41.9 \%)$ \\
\hline Stenosis \\
\hline Yes & $117(10.7 \%)$ \\
\hline No & $980(89.3 \%)$ \\
\hline Dilation & $89(8.1 \%)$ \\
\hline Yes & $1008(91.9 \%)$ \\
\hline No &
\end{tabular}

\section{Results}

During the study period, 1109 women undergoing saline infusion sonography were assessed for eligibility. Seven women refused to consent and were excluded. Of these 1102 women, 5 women got saline sonography under general anaesthesia and were excluded. A total of 1097 women were therefore included. Of these 1097 women, 117 had cervical stenosis. Eighty nine women agreed to have dilation and 28 women refused. Of these eighty nine women, two were lost to follow up, 1 from each group. These women were excluded from the analysis for conception. Figure 1 shows the study profile.

The participants had a mean age of $28.99 \pm 5.40$ years, a mean BMI of $27.57 \pm 4.39 \mathrm{~kg} / \mathrm{m} 2$ and a mean duration of infertility of $3.94 \pm 1.59$ years. Most couples had primary infertility. Of those included, $3.5 \%$ had history of endometriosis, $7.7 \%$ had previous episode of pelvic inflammatory disease, $5.4 \%$ had prior surgery and $6.6 \%$ had history of intrauterine polyps. Of those assessed at sis, 117 (10.7\%) had stenosis and were offered dilation. Table 1 shows characteristics and risk factors in the study population.

When stratified according to groups, both groups, those with stenosis and those without, were similar in mean age ( $p=$ $0.617)$, mean BMI ( $p=0.598)$, duration of infertility ( $p=$ $0.588)$ and type of infertility $(p=0.167)$. However, both groups were significantly different in terms of history for risk factors. Table 2 summarizes the comparison between the two groups.

Of the women who received dilation, $70.45 \%$ conceived as compared to $18.5 \%$ of those who had stenosis but refused dilation. The mean time to conception (in months) was also significantly lower in women who received dilation (8.10 \pm 3.43 vs. $11.39 \pm 1.97, p<0.001$ ) (Tab. 3 ).

Survival analysis also showed that the conception rate was significantly higher in those who opted to undergo dilation. (Log rank, $p<0.001$ ) (Fig. 2).

\section{Discussion}

\section{Main findings}

Our study showed that cervical stenosis existed in $10.7 \%$ of women with unexplained subfertility. History of endometriosis, pelvic inflammatory disease, prior surgery and polyps is significantly associated with cervical stenosis.

Cervical dilation in these women leads to better conception rates.

Tab. 2. Comparison of both groups in terms of history parameters

\begin{tabular}{|c|c|c|c|}
\hline \multirow{3}{*}{ Characteristics } & \multicolumn{2}{|c|}{ Stenosis } & \multirow{3}{*}{$p$-value } \\
\hline & Yes & No & \\
\hline & Mean \pm SD or count $n(\%)$ & Mean \pm SD or count $n(\%)$ & \\
\hline Age in years & $28.75 \pm 5.08$ & $29.02 \pm 5.44$ & 0.617 \\
\hline BMI & $27.36 \pm 4.48$ & $27.59 \pm 4.38$ & 0.598 \\
\hline Duration of infertility in years & $4.03 \pm 1.68$ & $3.93 \pm 1.58$ & 0.588 \\
\hline Type of infertility & $70(59.8 \%)$ & $567(57.9 \%)$ & 0.167 \\
\hline Primary & $47(40.2 \%)$ & $413(42.1 \%)$ & \\
\hline \multicolumn{4}{|l|}{ Secondary } \\
\hline History of endometriosis & & & $<0.001$ \\
\hline Yes & $17(1.7 \%)$ & $19(16.2 \%)$ & \\
\hline No & $963(98.3 \%)$ & $98(83.7 \%)$ & \\
\hline History of pelvic inflammatory disease & & & 0.001 \\
\hline Yes & $64(6.5 \%)$ & $21(17.9 \%)$ & \\
\hline No & $916(93.5 \%)$ & $96(82.1 \%)$ & \\
\hline History of surgery & & & $<0.001$ \\
\hline Yes & $39(4.0 \%)$ & $20(17.1 \%)$ & \\
\hline No & $941(96.0 \%)$ & $97(82.9 \%)$ & \\
\hline History of polyps & & & 0.004 \\
\hline Yes & $49(5.0 \%)$ & $23(19.7 \%)$ & \\
\hline No & 931 (95.0\%) & $94(80.3 \%)$ & \\
\hline
\end{tabular}


Tab. 3. Conception and time to pregnancy in women with cervical stenosis who received dilation vs those with no dilation

\begin{tabular}{|c|c|c|c|}
\hline & \multirow{2}{*}{\multicolumn{2}{|c|}{$\begin{array}{c}\text { Stenosis } \\
\text { Yes }\end{array}$}} & \multirow{5}{*}{$p$-value } \\
\hline & & & \\
\hline & \multicolumn{2}{|c|}{ Dilation } & \\
\hline & Yes & No & \\
\hline & $\begin{array}{c}\text { Mean } \pm \text { SD } \\
\text { or count } n(\%)\end{array}$ & $\begin{array}{c}\text { Mean } \pm \text { SD } \\
\text { or count } n(\%)\end{array}$ & \\
\hline \multicolumn{4}{|l|}{ Pregnancy } \\
\hline Yes & $62(70.45 \%)$ & $5(18.5 \%)$ & $<0.001^{*}$ \\
\hline No & $26(29.54 \%)$ & 22 (81.48\%) & \\
\hline Time to pregnancy & $8.10 \pm 3.43$ & $11.39 \pm 1.97$ & $<0.001^{*}$ \\
\hline
\end{tabular}

\section{Strengths and limitations}

Our study shows the importance of a full assessment of a couple before labelling them as having unexplained subfertility. Around $10.7 \%$ women had cervical stenosis and dilation led to conception in $70.54 \%$ of them. Another major strength is its case control design and extended follow up.

The major limitation of the study is the fact the single centre design; therefore generalizability of results in other populations needs to be verified with further larger multicentre trials.

\section{Interpretation}

Cervical stenosis is typically considered as a factor that may lead to infertility in women who have had surgery for cervical intraepithelial neoplasia $(\mathrm{CIN})^{(9)}$. However, it can also be a culprit in cases where no such history is present.
About $10.7 \%$ of women in our study had cervical stenosis, which is almost twice the figure reported in literature ${ }^{(2)}$.

Cervical stenosis is defined as a narrowing of the cervical canal, but no standardized diagnostic method is mentioned in literature. A recent study labelled women with stenosis if there was difficulty in passing a $3.5 \mathrm{~mm}$ sheath hysteroscope into the internal cervical os. We included women when it was difficult to pass a 7 French Foley's catheter ${ }^{(10)}$. Foley's catheter has a smaller softer tip that would theoretically include more women as having stenosis than when using a hysteroscope. We also argue that a hysteroscope is not accessible to all clinicians in outpatient departments and an opportunity would be missed in all such cases if the above criterion is used. A Foley's catheter is readily available and diagnosis is pretty straightforward with introduction into cervical canal.

A historical study showed that retrograde menstruation occurs when the external os is less than $4.5 \mathrm{~mm}$, which may lead to endometriosis and subfertility ${ }^{(11)}$. We would still argue that such a constriction may be notable to the woman and the clinician due to symptoms. If women were assessed for unexplained infertility any such history of retrograde menstruation would be conflicting. Furthermore, a 7 French Foley's has an outer diameter of $2.33 \mathrm{~mm}$, which is less than both the $3.5 \mathrm{~mm}$ hysteroscope and the external os diameter of $4.5 \mathrm{~mm}$. This cut-off needs to be assessed in further studies.

Because cervical stenosis is difficult to suspect and diagnose, many cases may go unnoticed until and unless the cervix is evaluated. This evaluation becomes more pressing in cases with certain risk factors. In their analysis,

Patients who were scheduled to undergo saline sonography

$N=1109$

Patients who consented and underwent sis in outpatient department

$N=1097$

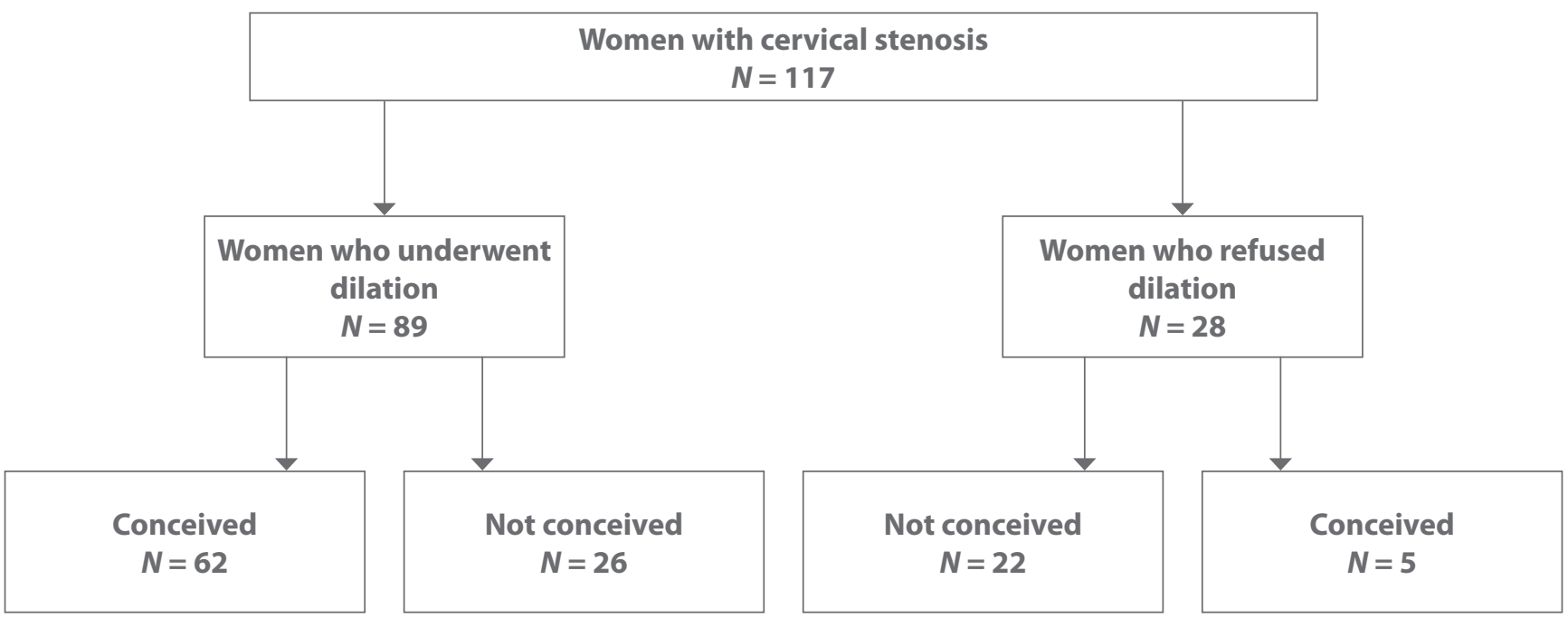

Fig. 1. Profile of the study 


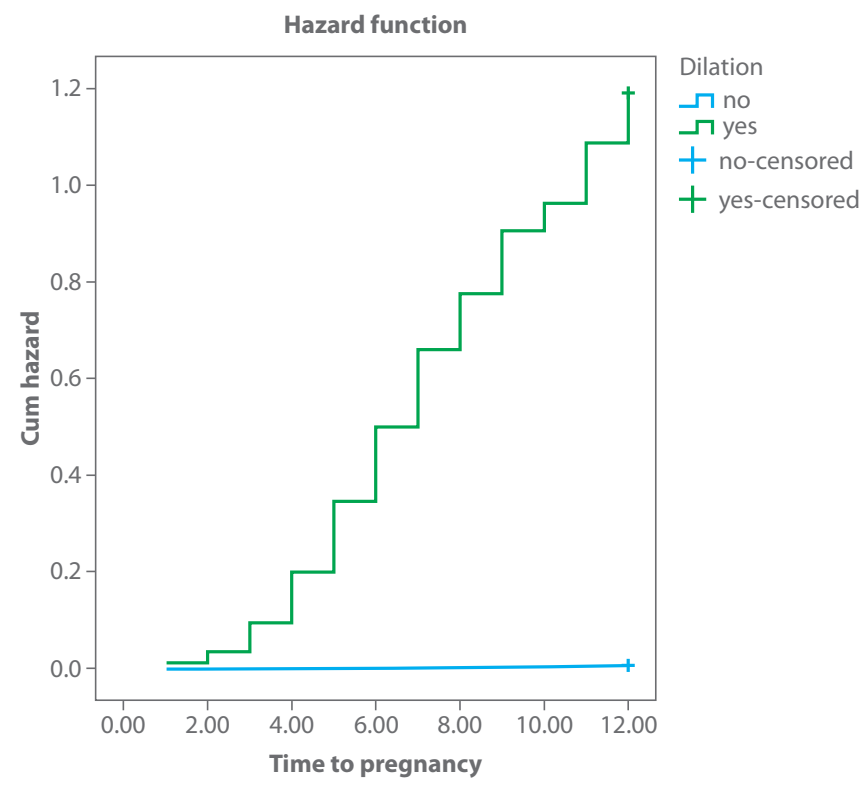

Fig. 2. Conception rate in both groups

Moramazi et al. showed a significant correlation with endometriosis, polyps and myomas ${ }^{(10)}$. In our study, prior history of endometriosis, reproductive tract surgery, polyps and pelvic inflammatory disease were significantly associated with cervical stenosis. These findings are in agreement with previous reports ${ }^{(12,13)}$.

It is important to diagnose and treat cervical stenosis. It hinders sperm deposition in both natural breeding and intrauterine transfers. False passages created during such iatrogenic intervention can be minimized by performing this intervention under ultrasound guidance ${ }^{(14)}$. We performed all dilations under ultrasound guidance. No false passages were created during the study.

Conception can increase after the cervical hindrance to sperm is removed. This has been the essence for adoption of intrauterine insemination in women with cervical factor subfertility ${ }^{(15)}$. Lin et al. demonstrated that cervical resection with hysteroscope in cases of stenosis improved conception at subsequent embryo transfer or intrauterine

\section{References}

1. Somigliana E, Paffoni A, Busnelli A, Filippi F, Pagliardini L, Vigano P, Vercellini P: Age-related infertility and unexplained infertility: an intricate clinical dilemma. Hum Reprod 2016; 31: 1390-1396.

2. Hull MG, Glazener CM, Kelly NJ, Conway DI, Foster PA, Hinton RA et al.: Population study of causes, treatment, and outcome of infertility. Br Med J 1985; 291: 1693-1697.

3. Kamath MS, Bosteels J, D’Hooghe TM, Seshadri S, Weyers S, Mol BWJ et al.: Screening hysteroscopy in subfertile women and women undergoing assisted reproduction. Cochrane Database Syst Rev 2019; 4: CD012856.

4. Carneiro MM: What is the role of hysteroscopic surgery in the management of female infertility? A review of the literature. Surg Res Pract 2014; 2014: 105412.

5. Edris FE: Is uterine depth measurement by trans-vaginal ultrasound alone as accurate as measurement carried out by trans-abdominal ultrasound-guided trial transfer?. Saudi Med J 2014; 35: 1231-1236. insemination ${ }^{(16)}$. In our analysis, women conceived spontaneously after dilation, and no intrauterine insemination or IVF was needed. This further cements the observation that cervical stenosis is a clinical entity that can be easily treated and women can conceive without further treatment.

The treatment modalities for unexplained subfertility are currently under scrutiny. Intrauterine insemination, ovulation induction and IVF have all been assessed in trials and currently no consensus exists ${ }^{(17,18)}$. In our study, a cervical cause was identified and the patients subsequently conceived after receiving dilation. It can be argued that this was not the cause of subfertility in these patients and a stringent protocol may have aided in ascertaining the actual cause. In response we would counter-argue that it may be the case that these women did not have unexplained infertility to begin with. Estimates for natural conception reported from a recent analysis show that women with unexplained subfertility have a $25 \%$ chance of conception even without treatment ${ }^{(19)}$. In our study, $70 \%$ of women who received dilation conceived compared to only $18 \%$ of the controls (who had stenosis defined as per criterion but refused dilation). This observation shows that such dilation is in fact curative and cervical stenosis does lead to subfertility. Natural conception rate of $18 \%$ in the control group also shows that in the absence of investigation for cervical factor these women would still have been classified as unexplained group and their conception rate was similar to that reported previously in larger trials ${ }^{(19)}$.

\section{Conclusions}

Our study shows that ultrasound-guided cervical dilation in women with cervical stenosis can lead to improved conception rates.

\section{Conflict of interest}

Authors do not report any financial or personal connections with other persons or organizations, which might negatively affect the contents of this publication and/or claim authorship rights to this publication.

6. Mahajan N, Gupta I: Use of Versapoint to refashion the cervical canal to overcome unusually difficult embryo transfers and improve in-vitro fertilization-embryo transfer outcome: A case series. J Hum Reprod Sci 2011; 4: 12-16.

7. Izhar R, Husain S, Tahir S, Husain S: Incidence of intrauterine abnormalities in Pakistani women with unexplained infertility diagnosed via saline infusion sonography. J Ultrason 2018; 18: 186-192.

8. Arora P, Mishra V: Difficult embryo transfer: a systematic review. J Hum Reprod Sci 2018; 11: 229-235.

9. Shah JS, Jooya ND, Woodard TL, Ramirez PT, Fleming ND, Frumovitz M: Reproductive counseling and pregnancy outcomes after radical trachelectomy for early stage cervical cancer. J Gynecol Oncol 2019; 30: e45.

10. Moramazi F, Roohipoor M, Najafian M: Association between internal cervical os stenosis and other female infertility risk factors. Middle East Fertil Soc J 2018; 23: 297-299. 
11. Barbieri RL: Stenosis of the external cervical os: an association with endometriosis in women with chronic pelvic pain. Fertil Steril 1998; 70: 571-573.

12. Fouda UM, Elsetohy KA, Elshaer HS, Hammad BEM, Shaban MM, Youssef MA et al.: Misoprostol prior to diagnostic office hysteroscopy in the subgroup of patients with no risk factors for cervical stenosis: a randomized double blind placebo-controlled trial. Gynecol Obstet Invest 2018; 83: 455-460.

13. Ahn SH, Monsanto SP, Miller C, Singh SS, Thomas R, Tayade C: Pathophysiology and immune dysfunction in endometriosis. Biomed Res Int 2015; 2015: 795976.

14. Kriseman M, Schutt A, Appleton J, Pillai A, George V, Zarutskie PW: A novel ultrasound-guided technique for hysteroscopic adhesiolysis in high-risk patients. J Ultrasound Med 2019; 38: 1383-1387.

15. Tjon-Kon-Fat RI, Bensdorp AJ, Scholten I, Repping S, van Wely M, Mol BW et al.: IUI and IVF for unexplained subfertility: where did we go wrong? Hum Reprod 2016; 31: 2665-2667.
16. Lin YH, Hwang JL, Huang LW, Seow KM, Chen HJ, Tzeng CR: Efficacy of hysteroscopic cervical resection for cervical stenosis. J Minim Invasive Gynecol 2013; 20: 836-841.

17. van Eekelen R, van Geloven N, van Wely M, McLernon DJ, Mol F, Custers IM et al.: Is IUI with ovarian stimulation effective in couples with unexplained subfertility? Hum Reprod 2019; 34: 84-91.

18. Tjon-Kon-Fat RI, Tajik P, Zafarmand MH, Bensdorp AJ, Bossuyt PMM, Oosterhuis GJE, et al.: IVF or IUI as first-line treatment in unexplained subfertility: the conundrum of treatment selection markers. Hum Reprod 2017; 32: 1028-1032.

19. van Eekelen R, Tjon-Kon-Fat RI, Bossuyt PMM, van Geloven N, Eijkemans MJC, Bensdorp AJ et al.: Natural conception rates in couples with unexplained or mild male subfertility scheduled for fertility treatment: a secondary analysis of a randomized controlled trial. Hum Reprod 2018; 33: 919-923. 